\title{
Les parcs d'attractions : jeu - divertissement - éducation
}

GillesBrougère

UniversitéParis-Nord

\section{Résumé}

Les parcs d'attractions se sont, dans les années 90, développés en France comme dans l'ensemble du monde. 1ls apparaissent comme des lieux de divertissement spécifiques dans la construction d'une illusion basée souvent sur des contenus culturels et/ou scientifiques sophistiqués. D'où la tentation d'y voir de nouveaux lieux d'éducation qui pourraient servir de modèles aux musées. Les expositions universelles sont des lieux qui mélangent volontiers des perspectives pédagogiques avec les procédés des parcs d'attractions.

L'article essaie d'analyser ce qui caractérise ce type de loisir et l'architecture qui lui est liée, proposant de nouveaux objets qui ressemblent à des jouets surdimensionnés tant leur logique est semblable. 11 importe en effet d'analyser la logique et le fonctionnement de ces nouveaux supports de loisir, pour comprendre leurs limites comme lieu d'éducation formelle. Celle-ci ne peut se développer que sur la base d'une analyse des parcs, d'une déconstruction de leurs effets.

\section{Mots chefs}

Parcs d'attractions - Divertissement - Loisir - lllusion. 


\title{
Amusement parks : play-entertainment-education
}

GillesBrougère

UniversitéParis-Nord

\begin{abstract}
The amusement parks grew in France, in the nineties, as they did all over the world. They appear as places dedicated to entertainment within the construction of an illusion based frequently on sophisticated cultural and/or scientific contents. Wherefrom comes the temptation of seeing in them new education places that could serve as models to the museums. The universal exhibitions are places that frequently mix pedagogic perspectives with procedures of amusement parks.

The article tries to analyze what characterizes this type of leisure and the architecture related to it, which proposes new objects that resemble oversized toys, since their logic is similar. It is indeed important to analyze the logic and the operation of these new leisure supports, to understand their limits as sites of formal education. This can only happen on the basis of an analysis of parks, of a deconstruction of their effects.
\end{abstract}

\section{Keywords}

Amu se ment parks-Enterta in ment-Le isure. 
La fin des an nées 80 et le dé but des an nées 90 vo i ent s'ouvrir en Fran ce le Fu tu ros cope, le Parc Asté rix, Euro Dis ney de ve nu de pu is Disneyland Paris. Aujourd'hui, face au succès de ces équipements, passés les problèmes rencontrés lors des pre miè res an nées, de nom bre ux pro jets de parc sont en cours d'élaboration, voire de construction. Ces attractions connaissent également de forts développements dans d'autres pays. Cette fin de siècle sem ble être ce lui du di ver tis se ment roi, où dévelop pementéco no mi que et tou ris ti que pas se par ces nouvel les in dus tri es du lo i sir, cet te mise en spec ta cle de no tre mon de. 11 faut ce pen dant ne pas se laisser abuser par les discours qui mettent en avant la totale nouveauté de tels lieux qui sont en fait les hé ri ti ers d'autres es paces dont ils re pren nent nom bre de ca rac té ris tiques. Nous pensons tout particulièrement à la fête fo ra i ne, mais on peut évo quer les jar dins ${ }^{1}$ et au tres fo li es à vo ca ti on dis trac tive.

\section{La structure du parc d'attractions}

Ces lieux semblent combiner trois composantes pour produire un divertissement ori ginal: le spectacle, le vertige ${ }^{2}$ et le jeu d'adresse. C'est le ver ti ge qui est le plus com munément associé à la fête foraine dont il cons ti tuel'emblème. Aujourd'hui des ma chi nes de plus en plus sop his ti quées créent des sen sa tions de vertige à ceux qui leur livrent leur corps. Mais le spectacle est également une composante traditionnelle, la base même et une des ori gi nes his to ri ques de la fête qui dressait ses tréteaux à côté de la foire. Spectacles extraordinaires, automates, raretés mais aussi premières ex ploi ta ti ons de tech niques et curiosi tésscien tifiques alimen tentle specta cle. Ain si le ci né ma y trou va un abri avant de gag ner son au to no mie. Enfin le jeu d'adresse ac com pag ne de telles manifestations sous des formes variées, avec ses lots à gag ner. Le stand de tir en estl'expression con tem po ra inela plus fré quente. Bien sûr chacune des composantes peut se développer séparément: le spectacle connaît bien des formes autonomes, le jeu d'adresse con naît un nou vel en gou e ment avec les jeux vi déo, et des sports pro po sent eux aus si et de plus en plus des expériences de vertige. L'originalité de la fête fo ra i ne c'est sans dou te l'association étroite de ces trois aspects qui dans certaines attractions tendent à devenir indissociables. Les formes changent et nous retrouvons ces trois composantes dans les parcs d'attractions. Ain si le parc Asté rix as so cie bien les tro is as pects, re pre nant d'ailleurs direc te ment des thèmes aux fêtes fo ra ines autour d'une présentation dans le style 1900.

C'est sans doute là que se distinguent les parcs Disney ${ }^{3}$ en développant très largement le spec ta cle (dont c'est la spé cia li té de son créa te ur) au dé tri ment du ver ti ge et sur tout du jeu d'adresse. En ef fet si le ver ti ge a mo ins de pla ce que dans la fête fo ra i ne et les parcs qui en dé rivent plus ou mo ins di rec tement, il est présent à travers d'importantes attractions, et semble devoir être développé en France pour répondre aux attentes de la clientèle européenne. En revanche, sous réserve d'inventaire, le jeu d'adresse disparaît ou devient marginal. A EPCOT (Orlando en Floride) on trouve quelques jeux vidéo et à Marne La Val lée (site de Dis ney land Pa ris), le stand de tir est la seuleattraction non com pri se dans le bil let d'entrée. En fait il faut ren vo yer ici à la ges ti on des parcs qui s'appuient sur un prix d'entrée qui don ne accès à tou tes les at trac ti ons. S'il est pos si ble de con trô ler le temps de pas sa ge sur un spec ta cle ou un jeu de ver ti ge, le jeu d'adresse peut in ci ter le jou e ur à con ti nu er le jeu, en ré du i sant d'autant

1. Les jardins furent des lieux de divertissement et de spectacles dont les parcs d'attractions, très attentifs à l'aspect paysager du site, gardent le souvenir.

2 Nous reprenons le terme utilisé par Roger Caillois (1967) dans Les Jeux et les Hommes, tout en contestant qu'il s'agisse là d'une forme de jeu.

3. II existe quatre parcs Disney, conçus sur le même modèle (Californie, Floride, Japon et France). 
l'accès aux au tres vi si te urs, saufà fa i re pa yer ce temps, ce qui est en con trac ti on avec le con trat d'accès à tou tes les at trac ti ons une fois la re de vance initialeréglée.

11 ré sul te de cela une fa i ble part ac cor dée au jeu dans de tels équipements. Nous contestons donc les analy ses qui dé fi nis sent par le ludi que les parcs (Lan quar, 1991). Elles s'appuient sur une définition vague du terme qui est pris comme un synonyme d'amusement. Le ludique a en gran de par tie dis pa ru dans le pas sa ge de la fête fo ra i ne aux parcs d'attractions, le sport ca pitalisant aujourd'hui une gran de par tie du ludi que adul te. En effet spec ta cle et ver ti ge im pliquent la passivi té duvisi te ur qui est en si tu a ti on d'être émer ve il lé ou se coué mais ja ma is d'agir à la façon du joueur: "Allégorie de la société de consommation, lieu de l'imaginerie absolue, Dis ney land est aus si le lieu de la pas sivi té to ta le" (Eco, 1985, p.73). Ce vi si te ur n'a pas d'influence sur le dé rou le ment de ce qui se pas se. 11 su bit et on pro du it sur lui des émo ti ons. Mais on at tend de lui l'obéissance à des injonctions ou des invitations pour être pro me né, as sis, se coué. Sa seule réac ti on est dans le cri lié au ver ti ge ou le murmure d'admiration face au spectacle surprenant. La parade qui permet d'assister à un dé fi lé tra du it bien la si tu a ti on duvisi te ur dansle parc. Un parc atypique, disparu pratiquement dès son ouverture (La Planète Magique) avait choisi à partir de thèmes proches des jeux de rôle, des livres dont vous êtes le hé ros et des jeux vidéo d'aventure, la stratégie interactive, celle $\mathrm{du}$ jeu, mais qui semble poser des problèmes tech ni ques dans le ca dre d'un parc qui doit maî tri ser le flux des vi si te urs et gé rer leur temps. Le spectateur passif est plus facile à gérer que le joueur.

L'équilibre entre les trois dimensions ca rac té ri se la fête fo ra i ne tout au mo ins dans son âge d'or de la fin du XIXe $\mathrm{e}^{4}$ siè cle, car aujourd'hui elle est mar quée par une di mi nu ti on im por tan te du spectacle concurrencé par l'industrie contemporaine du spectacle. 11 n'est pas étonnant alors que celle-ci, à partir de son savoir-faire, réa li se des parcs en réac tivant leur tra di ti on du spec ta cula ire. On peut aus si per ce vo ir le po int com mun en tre lever ti ge et le spec ta cle tel qu'il est conçu dans l'univers forain et peut-être plus encore dans les parcs d'attractions. L'un com mel'autre pro du it de l'émotion. Le spec tacle est construit plus pour ses effets que son con te nu en particuli ersousles for mes ci né matographiques nouvelles (3D, Omnimax, etc.) que l'on trou ve de fa çon mas si ve au Fu tu roscope, en complément dans d'autres parcs d'attractions. 11 n'est pas éton nant que se dé veloppe avec le cinéma dynamique un mixte entre la machine foraine et le spectacle. Le succès de cet équipement fait qu'aujourd'hui leFuturoscope en comptetrois.

Si le spec ta cle de vi ent lieu d'émotion et de vertige, parallèlement toutes les attractions participent à leur façon du spectacle: le ver ti ge ou l'adresse des au tres de vi ent spec tacle pour le vi si te ur. Par ail le urs les at trac ti ons sont le sup port de dé co ra ti ons qui tra du i sent souvent la mise en scène d'un thème. La dimension thématique, la mise en avant de l'image est un tra it tra di ti on nel de ce quel'on appelle aujourd'hui l'art forain. Chacun connaît ces manè ges qui évo quent un as pect de la réa li té ou de l'imaginaire. Ce qu'apporte

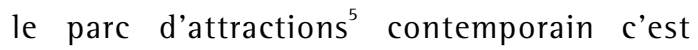
moins le thème que l'unité thématique de

4. C'est au XIXème siècle, avec l'apparition de nouvelles sources d'énergie, à commencer par la machine à vapeur que les manèges se développent et viennent compléter des fêtes vouées auparavant surtout au spectacle et à l'adresse. II s'agit du divertissement de l'ère industrielle par excellence: “Dans l'Angleterre des années 1870, le manège est pensé comme contrepoint de la machine agricole et de l'industrialisation" (Robichon, 1983, p. 324).

5. Nous préférons ce terme aux autres. Parc de loisirs est trop général et d'applique à d'autres équipements, parcs à thème s'applique difficilement. De plus parc d'attractions semble être utilisé largement, y compris par le grand public. II met l'accent sur le fait que ces parcs sont constitués du rassemblement d'attractions, qui peuvent être d'ailleurs les mêmes dans différents parcs, voire dans certaines fêtes foraines, parfois à I'habillage superficiel près. L'idée d'attraction renvoie bien au divertissement, à l'amusement (“Amusement parks" en américain), à la séduction de ces lieux. De plus ces parcs se conçoivent comme l'attraction touristique par excellence du lieu où ils s'implantent. 
l'ensemble de la fête, unité qui peut couvrir tout (ou pres que) le parc (Asté rix) ou une par tie du parc comme la décomposition chez Disney de l'espace en cinq régions porteuses d'un thème large comme l'aventure ou la conquête de l'Ouest. lly a là vo lon té d'une re la ti ve uni té thé ma ti que et une pré sen ce de l'image plus importan te, plusco héren te, plusspecta culaire. Le ou les thèmes deviennent le fer de lance pro motionnel du parc-spectacle. 11 s'agit de produire un espaceima gi na ire cré dible danslequel le visiteur-spectateur est pris. L'illusion doit être ma xi ma le d'où l'importance du tra va il sur le dé ta il, et l'échec de parcs qui ne sa vent pas tra vail lerà ce nive au, plon ger leurvi si te ur dans un uni vers complète ment étran ger à la vie quo tidienne(Mirapolis, Big Bang Schtroumpf). Le cas du Futuroscope est à part puisque l'ensemble thé ma ti que est as sez lâ che, au tour d'une évo ca ti on du fu tur, cha que spec ta cle gérant son pro pre système d'illusion.

A ceci va s'attacher un autre aspect qui dis tin gue net te ment le parc de la fête fo ra i ne, c'est la maîtrise et la délimitation de l'espace du parc: la sécurité, l'isolement (en particulier vi su el chez Dis ney de fa çon à ce que la vie or di naire ne pénètre pas sur le parc), le contrôle (dis cret mais effica ce), l'unité, la com mer ci a lisation des attractions globalisée par l'achat d'un tic ket qui don ne dro it à l'ensemble. Nous som mes dans un es pa ce to ta le ment maî tri sé où il n'y a rien à craindre. Tout ce qui arrive est con trô lé par la ma chi ne rie du parc. Au con tra ire, ou tre son as pect ép hémè re, la fête fo ra i ne tra di tion nelle est ca rac téri sée par un espa ceincer ta in (on ne sait par fo is où fini, où com men ce un es pa ce fon du dans la vil le), non con trô lé ou dont le con trô le n'est pas vi si ble, sans uni té, cha que fo ra in étant res pon sa ble de son at traction, où la violence semble pouvoir venir: l'image de la fête foraine est celle d'un lieu d'insécurité potentielle, exploitée par nombre de films. Mais il est vrai qu'il a exis té mas si ve ment jusqu'à la dernière guer re et qu'il exis te encore sous la forme de luna-parkprovisoires ouperma nents desespacesin termédia iresentre les deux équi pe ments. Dis ney a re pris très largement la tradition des Luna Parks et au tres Tivo li (Lan glo is, 1992), mais au jourd'hui certains exploitants n'hésitent pas à plagier les thèmes à succès des parcs Disney. On re trouve d'un lieu à l'autre les mêmes grands classiques (grand-huit, montagnes russes etc.) même si l'habillage varie.

La fermeture du parc, la construction d'un mon de ima gi na i re n'est pas sans ana logie avec l'utopie (Ma rin, 1973). Lieu de nul le part, le parc est l'espace du di ver tis se ment abso lu, par ce qu'en op po si ti on avecl'espace so cial quotidien. Et chez Disney cela s'étend à tou te une zone de lo i sir qui com prend hô tel et lie ux de diver tis se ment qui sont thé ma ti sés et ain si com me sor tis hors du mon de réel. A cet égard la notion d'architecture de divertissement ("Entertainment Architecture") développée par la société Disney est fort intéres san te. 11 s'agit d'une ar chi tec tu re figu rative qui règne dans le parc à tra vers la cons truction d'un décor adapté aux thèmes des attractions, mais également utilisée pour les hôtels dont chacun évoque une ambiance spécifique, comme le New York des années tren te, le Far West ou les grands parcs amé ri cains. Selon ses initiateurs, il s'agit de "se situer en pro lon ge ment del'expérience of ferte aux visiteurs par le parc à thème" (Euro Disney, 1992). Cet te ar chi tec tu re de diver tissement se développeaujourd'hui au bord de nom bre u ses rou tes sous for me de com mer ces divers, de restaurants ou d'hôtels. Messages et signes do minent une ar chi tec tu re qui doit convaincre au pre mi er coup d'œil.

\section{Thèmes et culture du parc}

11 en ré sul te que le parc est la cons truc tion d'une image qui devient essentielle; et sans fa ire pour au tant dis pa raî tre la fonc ti on, cet te ima ge joue un rôle cen tral pour con du ire le visiteur dans une ambiance spécifique. 
L'univers construit par Disney est semblable à un jouet maîtrisable par le visiteur: "L'imagination va ga bon de plus li bre ment avec un jou et", di sa it Dis ney ( L'architecture du ré confort, 1997). Nous sommes ainsi près de la lo gi que du jou et (Brougè re, 1989, 1992, 1995), où l'image prime souvent sur les autres aspects ${ }^{7}$. Le parc dis po se éga le ment de la tro i siè me dimension pour réaliser et crédibiliser ses représentations et utilise l'animation sous la for me, très fré quen te chez Dis ney, des au to mates. 11 s'agit de pro du i re des évo ca ti ons, des sig nifications qui entourent le visiteur pour le plon ger dans un univers his to ri que ou ima gi nai re. Mais à la diffé ren ce du jou et le plus sou vent minia tu risé, lesre présen ta ti ons sontà leur taille réelle, vo ire parfo is sur dimen si on nées; ets'ils sont réduits, ils doivent cependant donner l'impression d'avoir la ta il le réelle. Cela ex clut toute manipulation et en conséquence le jeu qui dérive de la possibilité de manipulation qu'offre le jouet. 11 s'agit pour le visiteur d'entrer dans une re pro duc ti on du mon de, non de la manipuler. C'est le spectateur qui à tra vers le spectacle et, surtout le vertige est un jouet manipulé par la machinerie et consentant, voireavide decet te ma ni pula tion.

Les thèmes mis en scène renvoient aux grands traits de l'imaginaire mais aussi à tra vers la mise en scène du fu tur à l'exaltation des sciences et des tech ni ques. A côté des con tes, de la ma gie et de l'aventure, la sci en ce trou ve ainsi sa place, aussi bien à travers l'évocation de Jules Verne, qu'à tra vers les der niè res nou ve a u tés de la tech no lo gie du spec ta cle. Un parc comme EPCOT est ainsi fondé sur la mise en scène des sci en ces et tech ni ques et le Fu tu rosco pesurla présen ta ti on specta cula ire destechno lo gi es de l'image (avec de nom bre ux films à contenu scientifique ou technologique). Le parc serait ainsi à rapprocher d'autres équipements com me le mu sée des sci en ces et tech niques. 11 semble avoir pour vocation de présenter à un large public des don nées de la science, les nou ve a u tés tech ni ques. Tel éta it le cas sur un thème réduit du parc Cousteau. Mais on peut se de man der ce qui est effective ment pré sen té. Plus que le mo yen de se re pé rer dans l'espace des sci en ces et tech ni ques il s'agit de trans for mer cel les-ci en spec ta cles, de provoquer enthousiasme et admiration, éven tu elle ment d'obtenir une ad hé si on àleur significa ti on so cia le. La sci en ce ou la tech nique ne sont que des élé ments cul tu rels par mi d'autres que le parc utilise pour construire son univers dis pa ra te: "En dé pit de leur thème af fi ché, le tra it sin gu li er des parcs est de juxtaposer des mondes disparates, au mépris de toute continuité diachronique et de toute continuité synchro ni que... d'où cet te im pression de bouillie culturelle" (Eyssartel et Rochette, 1992, p.75).

A be a coup d'égards le projet édu ca tif du parc est difficile à trouver. L'idée qu'il s'agit d'un espace pédagogique est souvent pos tulée sans que la mo in dre pre uve pu is se en être don née (Lan quar, 1991). Bien peu de moyens sont offerts au visiteur pour prolonger son im mer si on à tra vers une ré fle xi on ou des informations complémentaires. Les boutiques, au tra vers de mul ti ples gad gets, pro posent plutôt d'emporter chez soi une part de l'image qui a ravi le spec ta te ur. 11 s'agit là du sou ve nir in dis so cia ble du tou ris me de mas se. On cherchera va i ne ment à EPCOT com me au Fu tu ros co pe une libra i rie qui of fri ra it des ouvra ges de ré fle xi on, en co re mo ins une mé diathèque comme le font les musées.

Le parc trans for me la sci en ce et la technique en spectacle et propose au visiteur des émo ti ons, un choc af fec tif, une ad hé si on admi ra tive et non des ou tils d'information ou de réflexion. On trouve ra it pe ut-être quel quescas par ticu li ers con tra ires, mais la règle s'imposey com pris dans des parcs à pré ten ti on plus édu ca tive com me EPCOT ou le Fu tu ros co pe. C'est

7. Eyssartel et Rochette (1992, p.49) parlent d'un "monde jouet, à portée de main" et Eco (1985, p.65), "on ne pénètre pas dans une ville mais dans une ville jouet". 
vers la com mu ni on qu'ils pen chent. Et si le mu sée part d'un objec tif lui-même édu ca tif, il peut parfois lui empruntercertaines mi ses en scène. Res te que la diffé ren ce de me u re, il n'y a pas lieu de confondre trop vite ces équipements sous pré tex te que des mi ses en scènes pe u vent re poser sur la même tech ni que. L'objectif du parc est le divertissement, en utilisant tous les moyens pos si bles, y com pris ceux qui sont four nis par les sciences et techniques.

C'est sans doute là que l'exposition universelle contemporaine rejoint le parc d'attractions. A l'origine le projet est didactique et pro che de ce lui du mu sée, en chan ge ant l'orientation des œuvres du passé à celles du pré sent, vo i re de l'avenir. 11 s'agit de mon trer le progrès des arts et techniques. Et si la fête foraine ap pa raît, c'est en mar ge de l'exposition officielle, concession aux attentes d'un public qu'il im por te de voir se dé pla cer en mas se. Mais le programme officiel montre ce qui est, et suscite l'enthousiasme et l'admiration sur cet te base. Ce n'est que progressivement que le forain et la tech ni que vont se fon dre dans un parti pris de mise en scène spectaculaire des avancées de la science et de la technique ${ }^{8}$. L'espace de l'exposition doit à tout pris emporter l'adhésion du public. 11 s'agit d'une communion, parfois complexe, teintée de questionnement (tout au moins à partir de 1967 et de l'exposition de Montréal), dans le progrès ${ }^{9}$.

L'architecture, selon le principe repris dans les parcs, de vi ent en el le-même spec ta cle, image qui doit transmettre des significations au pu blic. Nom bre d'expositions res te ront célèbres pour leur re cons ti tu ti on de vil les ou vil la ges exo ti ques, des bâ ti ments du pas sé. 11 s'agit là en co re de fa ire naître une ad hé si on émo ti onnel le. Res te que si les mo yens sont sem bla bles, les objectifs de l'exposition universelle différent en ce qu'elles sont les moyens pour les États de fa i re con naî tre le urs réa li sa ti ons, leur dyna mis me, le urs tra di ti ons. L'architecture n'y est pas toujours figurative, elle est toujours significative d'autre chose que d'elle-même, au mo ins des com pé ten ces ar chi tec tu rales du pays. Nombre de pays ont abandonné l'architecture évo ca tri ce des tra di ti ons cul turelles, au pro fit d'une ar chi tec tu re con tem poraine aux accents futuristes à même de signifier l'engagement du pays dans la moder ni té. Le pa villon fran ça is de Sévil le 92 a été l'archétype d'une telle démarche qui accepte de sa cri fi er la qua li té et la ta il le des sur fa ces d'exposition au profit du geste architectural qui devient en lui-même l'essentiel de l'exposition. La Fran ce peut ex po ser du vide si ce vide est mis en scène de fa çon spec ta cu la i re par les ar chi tec tes. Ce n'est pas le con te nu oula fonction na li té du pa villon quiim portent mais l'image de la France qu'il propose. Ce pen dant le diver tis se ment n'est pas l'objectif fi nal de l'exposition uni ver sel le, c'est un moyen pour fa i re pas ser un mes sa ge plus po li ti que qu'éducatif. En cela il peut apparaître com me un objet noble. La der nière ex position du siècle, Hanovre 2000, si elle accorde une pla ce à quel ques at trac ti ons, s'engage dans la voie sérieuse de la réflexion sur le monde d'aujourd'hui tout en conservant la tradition desarchitec turesspecta culaires et desspectacles audiovisuelsimpression nants. Lepropos

8. Voir à ce sujet François Robichon (1983) qui montre comment, progressivement, l'exposition qui avait au début maintenu la fête foraine en marge, se fond progressivement avec cette dernière: "Enfin Osaka 1970 est une exposition gadget où la technologie architecturale est au service du fantastique et où de nombreux pavillons ne présentent que des attractions... Entre l'attractif et l'attraction, la frontière est ténue. Alors qu'en 1867 les distractions gravitaient autour d'un palais qui représentait l'ordre du monde, un siècle plus tard la structure dorsale d'Osaka est conçue par son architecte comme un espace de fête qui "exerce par lui-même une attraction". L'attractif est devenu la règle, l'attraction s'est banalisée en se généralisant" (p.318). Et plus loin: "Les Expositions universelles sont inséparables de leurs attractions. Si celles-ci ont constitué, à l'origine, un exutoire à des présentations industrielles trop didactiques, en prenant en charge les modes d'exposition et les contenus idéologiques, elles ont transformé I'Utopie en Attraction généralisée" (p.328).

9. "Les Expositions se transforment en une machine de propagande qui séduit plus qu'elle n'informe, faite sur mesure pour un public désireux de consommer un spectacle dans un espace aussi bariolé que dispersé" (Aimone et Olmo, 1993). 
pédagogique négocie avec le divertissement, mais af fir me une cer ta i ne vi si bi li té.

Ce n'est pas le cas du parc d'attractions dont il ne faut ja ma is ou bli er que l'objectif fi nal est de dis tra i re, tout le res te n'étant que moyens uti li sés en vue de cet te fin. En cela il est très pro che non seulement de la fête foraine qui n'a d'autre but, mais aus si de la té lé vi si on, au mo ins dans sa version commerciale.Soumis à la logique du divertissement, le visiteur, même s'il a d'autres objectifs, doit comprendre que cela a une in ci den ce sur l'ensemble du parc. Mais c'est aus si là que l'incompréhension ap pa raît avec les cri ti ques qui le plus sou vent se sont con cen trées sur Disneyland. Ainsi ce parc a été critiqué en tant que produit culturel marqué par le pays d'origine de ses concepteurs. Or nous croyons qu'il est bon de pré ci ser que Dis ney land n'a pas d'objectif cul tu rel, mais uti li se des élé ments culturels à des fins de divertissement. En conséquence les transformations du réel ou des sour ces ima gi na i res sont liées à ce deve nir divertissement qui permet d'attirer un large public qui ne vient pas pour rencontrer une culture quelle qu'elle soit, mais pour se divertir. On pour ra it dire à peu près la même cho se du ci né ma de diver tisse ment, de la télévi si on ou dujouet. Des éléments culturels, c'est-à-dire des objets do tés de sig ni fica ti ons, sont re pris, travaillés pour en faire des produitsindustriels pour lesquels la finalité(diver tissement)l'emportesur la signification ${ }^{10}$. S'il y a une critiqueidéologique à fa i re c'est mo ins dans les con te nus qui fi nale ment im portent peu que danslava lo risa tion $\mathrm{du}$ divertissement comme finalité dernière de no tre mon de. A cet égard l'impact de la té lé vi sion est autrement plus for te que cel le des parcs d'attractions (Post man, 1986). Res te que la cri tique semble porter sur la légitimité même du divertissement anodin (opposé au loisir noble, occasion de s'éduquer). Le divertissementest-il lé gi ti me? N'a-t-il pas de pu is tou jours fait feu de tout bois, c'est-à-dire uti li sé de mul ti ples sour ces culturelles pour nourrir ses scénarios. L'industrie du divertissement est rarement créatrice; elle utilise les sources disponibles pour les trans for mer, et Dis ney a été un maî tre en la matière, même s'il a eu aussi une phase créatrice. Car si le divertissement utilise des contenus culturels préexistants, il lui arrive d'en créer de nou ve au ou de pro po ser des combinaisons originales d'éléments antérieurs. N'oublions pas que Mo liè re se si tu a it dé li bé rément dans une pratique de divertissement com me nom bre de créa te urs cul tu rels.

$11 \mathrm{im}$ por te de pren dre ces pro du its pour ce qu'ils sont. Des instruments de divertissement, qui doivent permettre au visiteur de pren dre pla i sir à ce qu'il voit et vit. Et il sem ble que l'illusion est essentielle à cet investissement du vi si te ur-spectateur. Com me la fic ti on d'un ro man ou d'un film tra di ti on nel, le parc ne mon tre pas ses cou lis ses, sa ma chi ne rie: "A la diffé ren ce des vil les, le 'tra va il' qui as su re le fonc ti on ne ment des parcs doit res ter in vi si ble en tant que tra va il: il doit se dis si mu ler, com me dans les couloirs souterrains des parcs Disney, les 'uti li dors', ou se dé gui ser en ani mation, mieux encore être une animation" (Eyssartel et Rochette, 1992). 11 doit donner l'impression d'une na tu ra li té là où, bien sûr, il y a une construction ar tificielle. C'est un lieu d'oubli où tout rap pel du mon de réel est tra qué com me des truc ti on de l'ambiance. Là en co re Dis ney, avec son at ten ti on aux plus pe tits détails, est maître en la matière. En d'autres temps, on au ra it par lé d'opium du pe u ple. 11 ne s'agit pas pour nous de re fu ser sa lé gi ti mi té au diver tis se ment. Au nom de quoi re fu ser le di ver tisse ment aussi sop his tiqué so it-il. ll est des il lu si ons plus dan ge re u ses que cel les qui n'ont d'autres ob jec tifs que ce lui de diver tir.

10. Eyssartel et Rochette (1992); soulignent bien la continuité culturelle de la télévision et du parc: "La télévision peut être considérée comme la propédeutique à la pratique du parc. Le parc à thème est la spatialisation d'un monde connu que le visiteur 'reconnaît' parce qu'il en possède la grille de lecture. La télévision en lui délivrant ses contenus et leur syntagmatique où la contamination généralisée des genres est la règle, et la coprésence de mondes, en une télétopologie mentale, la quintessence, lui a fourni l'instrumentalité des parcs" (p.75). 


\section{Parc, divertissement et projet éducatif}

Se lon Eli as et Dun ning (1994) les ac ti vi tés de lo i sir ont pour fonc ti on de com pen ser le con trô le des af fects et émo ti ons que la so cié té moderne liée au processus de civilisation deman de auxin divi dus. Pour ce fa ire el les pro posent des activités mimétiques qui permettent de sus ci ter des émo ti ons de ve nu es lé gi ti mes. 11 perçoit ainsi trois éléments essentiels aux loisirs: “la so ci a bi li té, la mo bi li té et l'imagina tion” (p.164). La sphère du lo i sir de di ver tis se ment doit donc proposer aux individus des activités libres, mimétiques, sans danger ni risque (parce qu'elles sont fictives et non réelles), per met tant l'excitation et l'expression de l'émotion dans un temps et un es pa ce dé li mité, compatibles avec l'autocontrôle que requi ert no tre so cié té. Si Eli as n'évoque pas, à ma connaissance, le parc d'attractions mais s'intéresse plus particulièrement au sport, on peut percevo ir com bi en le parc ré pond de fa çon pré ci se aux critè res d'Elias. 11 s'agit bien de produire de l'émotion à travers de la réalisation d'un espace mimétique dans la mesure où l'illusion y est essentielle avec le recours à la tro i siè me di men si on qui ins ère le public dansle mon deima ginaire. Les procédés cinéma to graphi ques du Fu tu ros co pe ont ain si pour ca rac téristiquedominante de dé pas ser le côté fron tal et dis tant de l'image du ci né ma or di na i re pour fa i re en trer le spec ta te ur dans l'image avec divers procédés fort efficaces comme le cinéma circula ire, l'Omnimax ou le ci né ma dyna mi que. L'excitation est éga le ment pré do minan te de façon à ce que le pu blic vive des émo ti ons de façon cré di ble, mais dans un es pa ce qui ap pa raît com me fic tif et donc sans ris que. L'illusion est bien le fac te ur do mi nant de la cons truc ti on du di ver tis se ment dans la me su re où il per met de produire une excitation et des émotions qui sont, si l'on suit Eli as, la jus ti fi ca ti on même du loisir ou du divertissement. A cela on peut ajouter l'importance de la mise en scène de la so cia bi li té fon da men ta le dans les parcs qui se veulent des lieux de convivialité même si là en co re l'illusion l'emporte sur le réel. 11 s'agit bien de communier à travers des émotions communes. Enfin la motricité, même si elle est largement mécanisée, est constitutive d'une telleexpérience.

Notre question porte alors sur l'utilisation d'un ob jet fait pour le di ver tis se ment dans un ca dre édu ca tif. Ne nous oc cupons pas de l'enseignant qui choisit le parc com me lieu de diver tis se ment de fin d'année, il ne de vra it pas avo ir be so in d'alibi édu ca tif. Et l'expérience que nous en avons mon tre que même des étu di ants con cer nés parle do ma ine et sensés prendre une distance critique s'enfoncent aisément dans le divertissement, l'illusion et le pre mi er de gré. Mais sans dou te est-ce une ex péri en ce in con tour na ble. 11 faut sacrifier au plaisir, au divertissement, à l'illusion avant de son ger à tou te au tre utili sa ti on du parc d'attractions. Le spec ta cle proposé, contrairement à celui qui porte des significations qui en constituent sa fin, n'a d'autre ho ri zon que lui-même et doit pro poser au spectateur un univers qui se suffit à lui-même: "La dis tan ci a ti on, qui fon de la rela ti on spec ta cula ire, dis pa raît au pro fit d'une immersion dans le spectacle lui-même où le spectateur participe à la mise en scène. 11 se re trou ve au cœur de l'espace scé ni que non seu le ment par le re gard mais par une synest hé sie totale avec le spectacle" (Eyssartel et Ro chet te, 1992, p.79).

Au-delà est-il pos si ble d'utiliser le parc en fonction d'objectifs éducatifs? C'est là qu'il con vi ent d'être vi gi lant. Cer tes les parcs proposent des documents pédagogiques, mais il n'est pas dit que pour au tant ils ai ent changé d'objectifs et que tout d'un coup, ils so i ent devenus des es pa ces édu ca tifs. Bien sûr ily a là des con te nus cul tu rels ri ches et va riés, mais dont il faut savoir qu'ils sont tous pré sen tés sur le même ni ve au quel que soit leur origine, mêlant sans les distinguer 
l'imaginaire et la vé ri té his to ri que plus ou mo ins mo di fiée, le vrai et le faux, tout cela étant soumis à l'impératif du divertissement et de l'illusion ce qui n'est pas en tant que tel il lé gi time. On ne peut faire comme si tout d'un coup, on pou va it pren dre les mê mes con te nus et les con si dé rer com me sig ni fiants d'un po int de vue édu ca tifs. lls ont été trans for més, tra vaillés pour plaire, séduire, produire de l'émotion, de l'illusion", non comme support de connaissance.

Or l'illusion ${ }^{12}$, la re la ti on émo ti on nel le à un con te nu im pli quent un état d'esprit an ti nomi que au projet édu ca tif. En par ti cu li er la fu sion in ter dit la dis tan ci a ti on qui rend pos sible la réflexi on. 11 se ra it il lu so i re de pen ser que la fréquen ta ti on du parc en tant que tel le per met te d'apprendre quoi que ce soit. Tout au plus pe ut-on ima gi ner pê cher ici ou là une in for ma tion (Est-elle vra ie? Est-elle fa us se?), ren con trer une émo ti on qui in vi te à al ler plus loin après, ce que l'on appelle "être sensibilisé à".

D'où la direction que nous pro po sons: il n'y a pas d'usage éducatif possible du parc d'attractions sans une prise en compte de ce qu'il est, de la fa çon dont il fonc ti on ne. 11 faut cas ser l'illusion ${ }^{13}$, sor tir du cer cle de la sé duc ti on, met tre sur la ta ble les prin ci pes de fonc tionnement pour pouvoir utiliser ensuite les éléments culturels disponibles, en ayant toujours à l'esprit qu'ils sont pré sen tés en fonc ti on d'objectifs spécifiques qui en transforment le sens. Pe ut-être la tâ che est-elle plus fa ci le dans le Parc Astérix dont le ré fé rent est déjà un jeu sur l'illusion et in cor po re le se cond de gré. Tous les parcs sont-ils prêts à ac cep ter ce dé tour par les coulisses, cette déconstruction préalable à un vé ri ta ble usa ge édu ca tif du parc? Rien n'est moins sûr. D'où l'importance de la formation de l'enseignant qui doit disposer des outils pour com pren dre, re si tu er les con te nus et ain si est-il pos si ble de fa i re un tra va il au tour des éléments cul tu rels four nis par le parc sans ja ma is les pren dre au pre mi er de gré, pas sé le né ces sa ire moment d'acceptation de l'illusion qui devient utile en ce qu'elle confère à chacun une ex pé ri en ce qu'il peut analy ser dans un second temps.

Pour objectiver ce tra va il sur le parc, il est im por tant de pas ser par une con na is san ce du système tech nique quile pro du it: ma chine ri es en tout gen re, ges ti on des flux de vi siteurs, techniques cinématographiques... Il im por te de com pren dre la ma chi ne parc et ensu i te son produ it pour pouvo ir utiliser la vi site d'un point de vue éducatif. C'est toujours situer le parc com me une ma chi ne à di ver tir, mais après il est sans dou te pos si ble d'utiliser l'émotion, l'intérêt des élè ves pour une ré fle xi on sur ce qui l'a pro du i te et sur les élé ments cul tu rels uti li sés pour cela. Bien sûr un tel travail prend des formes différentes selon l'âge desélè ves, et la pé da go gie d'une tel le dé marche res te en co re à gran de par tie à in ven ter. 11 nous paraît illusoire d'utiliser les éléments culturels des parcs en oubliant le contexte dans le quel ils s'insèrent et les fi na li tés spé cifiques qu'ils portent.

11. "Je pense que, dans ce genre de parc, la disparition de l'incrédulité dépend directement du talent avec lequel l'illusion est créée en toile de fond." Propos de Tony Baxter, vice-président, responsable de la création de Disneyland ( Euro Disney ,1992, p.68) 12. L'architecte Paul Chémotov, Iors d'une présentation publique de son travail pour la rénovation de la Grande Galerie du Muséum d'Histoire Naturelle a pu dire en qualifiant le parti pris de mise en scène muséographique: "L'allusion pas l'illusion" avec comme exemple les singes sur des structures et non de faux arbre, les animaux arctiques sur du verre et non de la fausse glace. II s'agit d'un refus du faux aux implications pédagogiques et culturelles évidentes. Mais l'objectif du Muséum n'est pas le divertissement, même s'il n'est pas interdit de le fréquenter pour se distraire.

13. A cet égard le Cinémax (cinéma dynamique) du parc de la Villette à Paris était, dans sa première version, tout à fait intéressant: après la séance productrice de l'illusion kinesthésique associée au film, une vitre permettait de voir la machinerie fonctionner pour la séance suivante et donnait donc la clef technique. Mais pour certains il pouvait s'agir là du spectacle supplémentaire d'un monstre métallique en mouvement. II est vrai que cette salle se situe en relation avec la Cité des sciences et doit donc justifier son existence dans ce contexte où les objectifs éducatifs l'emportent sur les autres. 


\section{Références bibliographiques}

LA TOUREiffel etl'exposition uni ver sel le. In: RÉUNI ONDESMUSÉESNATIONAUX, Pa ris, 1989.

AIMONE, Lin da; OLMO, Carlo. Lesexpositionsuniverselles, 1851-1900. Pa ris: Be lin, 1993.

L'ARCHITECTURE du ré con fort: les parcs thé ma ti ques de Dis ney . Mon tréal: Cen tre Ca na di en d'Architecture, 1997.

BROUGĖRE, Gilles. Lare présentation de l'habitatdans le jou et . Le Jouet: revue autrement. Mutation, n¹33, nov. 1992. Brinquedo e cul tura. São Pa u lo: Cor tez, 1995.

CAILLOIS, Ro ger. Les jeux et les hom mes. Pa ris: Gal li mard, s.d.

DUMAZEDIER, Jof fre. Versune civilisation duloisir. Pa ris: Edi ti ons du Se u il, 1962.

ECO, Umber to. La guer re du faux. Pa ris: Gras set, 1985.

ELIAS, Nor bert; DUNNING, Eric. Sportetcivilisation, laviolence maîtrisée. Pa ris: Fa yard, 1994.

EURODisney. ConnaissancedesArts, 1992. Nu mérospécial.

EYSSARTEL, Anne-Marie; ROCHETTE, Ber nard. Desmon des in ven tés: les parcs à thème. Pa ris: Édi ti ons de la Vil let te, 1992.

LANGLOIS, Gilles-Antoine. Jours de fête: de Tivo lià Eu ro Dis ney land. Pa ris: Syros, 1992.

LANQUAR, Ro bert. Les parcs de lo i sirs. Pa ris: P.U.F, 1991.

MARIN, Lou is. Uto piques, jeux d'espace. Paris: Editi ons de Mi nu it, 1973.

ORY, Pas cal. LesexpositionsuniversellesdeParis . Pa ris, 1982.

PAYSAGE: parcs urbains etsuburbains. Cahiers du C.C.I. Pa ris, 1988.

PINOTDEVILLECHENON, Florence. Lesexpositionsuniverselles. Pa ris: P.U.F, 1992.

POSTMAN, Neil. Se dis tra ireà en mou rir . Paris: Flam marion, 1986.

REBÉRIOUX, Ma de la ine. "Les ou vriers etles expo sitions univer sel les de Pa ris auXIXe siè cle" In: LELIVREdes expositions universelles, 1851-1989. Pa ris: Edi ti ons des arts dé co ra tifs, 1983.

ROBICHON, Fran ço is. "L'attraction, pa rer gon des ex po siti ons uni ver sel les". In: LE LIVRE des expositionsuniverselles, 1851-1989. Pa ris: Edi ti ons des arts dé co ra tifs, 1983.

SUE, Roger. Le lo i sir. 2. ed. Pa ris: P.U.F., 1993.

Rece bi do em 24.01.2001

Apro va do em 22.03.2001

Gil les Brougè re é pro fes sor da Uni ver si da de Pa ris-Nord e di re tor ad jun to do Groupe de Recherche et Etudes Culturelles (GREC). Co or de naum Cur so de Espe cialização em Ciên cias do Jogona Uni ver si da de Paris XIII. 